\title{
Is there a Difference in Cognition between Epileptic Subjects and Healthy Siblings?
}

\author{
Gien-Lopez JA ${ }^{1,2}$, Hernandez-Rodríguez HG $^{1}$, Castillo-Ibarra EH ${ }^{2}$, Martinez-Cerda $\mathrm{G}^{1}$, Guerrero-De Leon \\ $\mathrm{JE}^{1}$ and Rodriguez-Leyva I*1,2 \\ ${ }^{1}$ Facultad de Medicina de la Universidad Autonoma de San Luis Potosi, Mexico \\ ${ }^{2}$ Neurology Service, Hospital Central “Dr. Ignacio Morones Prieto", Mexico
}

*Corresponding author: Ildefonso Rodriguez Leyva, Neurology Service, Facultad de Medicina de la Universidad Autonoma de San Luis Potosi, Hospital Central "Dr. Ignacio Morones Prieto", San Luis Potosi, SLP, Mexico.

Received Date: August 05, 2019

Published Date: August 09, 2019

\section{Abstract}

Objective: To compare the cognitive status of epileptic patients diagnosed with focal epilepsy with unknown etiology (FEUE) or juvenile myoclonic epilepsy (JME) compared to a healthy brother or sister and to correlate this cognitive status to the drug treatment administered (monotherapy or polytherapy) and the time of diagnosis of epilepsy ( 9 or fewer years or 10 or more years).

Material and Methods: An analytical, case-control, cross-sectional, descriptive, observational study was conducted to determine the cognitive status of patients with the diagnosis of FEUE or JME compared to a healthy sibling using the Community Scale Interview for Dementia (CSID). The data was captured in the Excel software to obtain results and analysis of all the items of the CSIDs that included orientation, memory, praxis, attention and calculation, and language. The sample was taken for convenience during November 2010 in the Neurology consultation of the Central Hospital "Dr. Ignacio Morones Prieto" in San Luis Potosi, Mexico. A total of 30 epileptic patients and 30 healthy siblings were included.

Results: 30 couples were studied, consisting of a patient diagnosed with FEUE or JME and a healthy control brother or sister with a + - 3 years difference in their age. The CSID results showed a difference of 1.7 in the language area in favor of healthy subjects, but differences of $-0.13,-0.07$ and -0.02 in the areas of memory, orientation and praxis, respectively. The analysis based on the treatment the patient had shown no statistically significant differences, supporting the hypothesis that there would be no difference in the cognitive items evaluated by the. The analysis according to the time of diagnosis pf epilepsy obtained $\mathrm{p}>$ of 0.05 , reflecting that there are no differences in the cognitive status regardless of the time of diagnosis.

Conclusion: There was no statistically significant difference in the areas of memory, praxis, language, orientation, attention and calculation when comparing the epileptic patient with FEUE or JME with a healthy brother regardless of the type of treatment received (monotherapy or polytherapy) or the evolution time since the diagnosis of epilepsy ( 9 or less years against 10 or more years).

Keywords: Focal epilepsy with unknown etiology (FEUE); Juvenile myoclonic epilepsy (JME); Community-Scale interview for dementia (CSID); Cognitive status

\section{Introduction}

Epilepsy affects between 1 and 2\% of the Mexican population and is a controllable neurological disease if a timely diagnosis, proper management, and adequate treatment adherence are made. Acquired epilepsy is a frequent problem in developing countries, and the most common cause is by infectious agents, being the cysticercus the leading cause in our country, in addition to the worldwide known etiologies, as the cerebral vascular event, trauma, and tumors. Within the different types of epilepsy, two are of particular interest for this study: a) Focal Epilepsy with Unknown Etiology (FEUE), defined as one in which there is an abnormal paroxysmal focal discharge in which there is no evidence of structural, genetic, infectious, metabolic or immune etiology, or other symptoms or signs for another neurological disease, b) Juvenile Myoclonic Epilepsy (JME), defined as generalized epilepsy characterized by myoclonus, absence and/or generalized seizures. JME has a multifactor genetic background that has not yet been fully established.

Patients with epilepsy suffer stigmatization in terms of their cognitive abilities, which often causes them to be relegated from school, work, and domestic activities by family, teachers, and 
workers. This situation economically impacts the patient with epilepsy, but it also impacts the patient's family.

Several studies have been given the task to establish a relationship between epilepsy per se and cognitive impairment. However, most of these studies aimed at demonstrating this deterioration based on the time with the diagnosis of epilepsy, especially based on the pharmacological management received. Some studies have carried out of cases and controls groups for this same purpose; however, no work has ever compared the cognitive state of the patient with epilepsy against the cognitive state of a healthy brother.

Some reports indicate that patients diagnosed with FEUE and JME have disturbances in short-term memory, language, attention and calculation, and praxis when analyzed as a cases and controls group. In studies that have evaluated epileptic patients according to the drugs they used, similar results have been found in patients using first-generation drugs such as Valproate, Carbamazepine, and Phenytoin, as well as second-generation ones such as Levetiracetam and Lamotrigine. Another approach to the cognitive state of this patients is the analysis based on the time of evolution of the seizures, where significant differences have been found in patients with more than ten years of evolution of epilepsy compared with those who have less than ten years of being diagnosed.

In order to carry out this research, the Community Scale Interview for Dementia (CSID) scale was used to assess the memory, language, praxis, care, and calculation. This scale had already been used for assessment in epileptic patients in a study developed in Nigeria. This study was conducted in 30 pairs of siblings, one diagnosed with FEUE or JME, and a healthy brother. The results of the qualification obtained in all the items were analyzed, making the first analysis by pairs of siblings and later as groups of cases and controls. The analysis was also focused on medical management such as monotherapy or polytherapy and by the time of evolution. The results showed that in the subjects studied, there was no statistically significant difference in any of the items assessed by the CSID. Based on these results, it is possible to propose the dissemination of research work in the general population and to the health authorities to implement the necessary strategies to reduce the stigmatization of the patient with FEUE and generalized myoclonic epilepsy, since the patient the same cognitive skills as a healthy brother.

\section{Background}

In 1973, the International League against Epilepsy (ILAE) and the World Health Organization (WHO) defined epilepsy as the chronic recurrent condition of paroxysmal crises, triggered by abnormal electrical discharges that have varied clinical manifestations of multifactorial origin and are associated to paraclinical disorders such as electroencephalographic abnormalities that occur in an unprovoked manner. By definition, epilepsy is diagnosed after a patient has had two or more unprovoked seizures [1]. Regarding the epidemiology of epilepsy, most of the studies are categorized as: those carried out in developed countries and those carried out in developing countries. The difference between these countries is based on socioeconomic criteria and level of industrialization of each region. For practical purposes, developed countries are those of Europe, North America, and Australia. Developing countries are those in Central and South America, as well as those in Africa and Asia. In developed countries, Europeans report prevalence ranging from 6.2 to 7.6 per 1000 population; in children, it is generally lower with a range of 3.6 to 5.3 per 1000 . There are North American reports of 6.8 per 1000 in adults and 4.7 in children, so the prevalence is similar in both regions. In developing countries, there are significant differences between the prevalence reported between one country and another. For example, in South America, higher prevalence is reported: in Chile 17 per 1000, Bolivia 10 per 1000, in African reports it is estimated 5.2 per 1000 inhabitants, and in Asia, mainly India 5.3 per 1000 [2].

In Mexico, in studies conducted between 1983 and 2002 reported a prevalence of 10.8 to 20 per 1000 inhabitants [3]. Based on gender, most studies show a higher prevalence of men over women, but no proportion has been established for this since Latin American studies have shown a slightly higher prevalence in the female gender [4]. It is estimated then, that worldwide there are between 40 and 50 million patients diagnosed with epilepsy in their different varieties and that is why the WHO, in 2001, accepted that epilepsy means a Public Health problem due to its magnitude, significance, and vulnerability.

The significance of this problem is notorious since it affects the whole society considering that epilepsy is a chronic condition, with high morbidity and low mortality. Epilepsy vulnerability refers to impact measures to prevent and also control the factors involved in the genesis of epilepsy. It is known that the main risk of acquiring epilepsy are perinatal disorders, particularly neonatal anoxia, head trauma, neurocysticercosis, and cerebral vascular events (CVD). National authors have concluded that in Mexico, the prevalence of epilepsy fluctuates between 1 and $2 \%$, with higher values than those referred to in international literature.

Epilepsy has been classified on different occasions by the International League of Epilepsy, since its creation in 1960, being the last update in 2017 The new Classification of the Epilepsies is a multilevel classification, designed to cater to classifying epilepsy in different clinical environments. This is in acknowledgment of the wide variation in resources around the world, meaning that different levels of classification will be possible depending on the resources available to the clinician making the diagnosis. Where possible, a diagnosis at all three levels should be sought as well as the etiology of the individual's epilepsy. The starting point of the new Epilepsy classification framework is the Seizure Type; it is determined according to the new nomenclature into focal onset, generalized onset, and unknown onset. The second level is that of Epilepsy Type. It assumes that the patient has a diagnosis of epilepsy based on the 2014 definition and includes a new category of "Combined Generalized and Focal Epilepsy" in addition to the 
well-established Generalized Epilepsy and Focal Epilepsies. It also includes an Unknown category. The third level is an Epilepsy Syndrome diagnosis, referring to a cluster of features incorporating seizure types, EEG, and imaging features that tend to occur together [5].

Epilepsy can affect the quality of life of patients, in different areas, one of them is the stigma that causes the disease that patients with epilepsy may have a cognitive impairment, less professional qualities, and in general risk for people who surround the patient. There are also different myths, misconceptions, and stereotypes concerning epilepsy that are even documented throughout history. These myths are prevalent in society and in particular in individuals who have not had contact or known someone with this condition, the most common examples are that patients with epilepsy have poor academic performance, lower work capacity and can be a danger to companies and their insurers [6-13]. These stigmas of rejection and discrimination negatively affected the number of years in school in patients who recognized being tried for epilepsy in a study conducted with 445 people with epilepsy in the United States [14-20].

The relationship between epilepsy and cognitive function appears to be bidirectional. Research has shown that cognitive dysfunction can precede the onset of seizures in children. It has also been shown that children with a recent diagnosis of epilepsy have, by the time of the onset of seizures, deterioration in intelligence, executive functions, language speed, and psychomotor measures. Multiple variables that impact cognitive function have been related, including the type of crisis [21-24], the frequency of epilepsy [10], age of onset [9], etiology [25-26], duration of epilepsy [27], and the type and number of antiepileptic medications used [28].

In this regard, the temporal lobe epilepsy is one of the less likely to be associated with intellectual abnormalities, at least compared to birth disorders and epileptic syndromes. Temporal lobe epilepsy, mainly when it is bilateral, is commonly associated with problems with language, verbal memory, or post-natal psychotic episodes. However, unilateral epilepsy causes minimal problems that can affect the individual negatively or in academic performance and daily life [29]. Temporal mesial epilepsy is the most frequent focal epilepsy and is characterized by having hippocampal sclerosis and for being resistant to pharmacological antiepileptic management. The range of cognitive functions that this type of epilepsy can compromise include intelligence, learning, spatial-visual functions, memory, and attention [30,31]. In a study in epileptic patients in Nigeria using monotherapy with first-generation drugs, we have found that there is no statistically significant difference in cognitive tests in patients using phenytoin or carbamazepine [32-34]. As for the new generation antiepileptics, those who appeared after 1990 have been associated with adverse effects; however, not specifically related to cognitive functions. Of these, lamotrigine stands out, which has been related to mood swings but without causing an alteration in cognitive functions compared to placebo and even valproate [35].
For the assessment of cognitive functions in epilepsy, different instruments have been used that evaluate the areas of language, memory, attention, orientation, and calculation, these being the most affected fields as described in this document [16,17,22,26,2839]. Most researchers use the Folstein's Mini-Mental or cognitive skills instrument. These scales have been synthesized in a community dementia screening interview, called Community-Scale Interview for Dementia, which has already been validated in our country $[40,41]$, moreover, tested in different populations around the world, such as Korea [42], Nigeria [34] Canada, the United States, and Jamaica [43]. The CSID evaluates the use of language, memory, attention, calculation, and praxis to the examiner. It was developed for screening in cross-cultural studies and consisted of components for populations without education, and with formal education, it has demonstrated the adaptability and usefulness in different populations with different socioeconomic levels in addition to having sensitivity and specificity higher than the cognitive scales alone [43]. The results of studies of cognitive level and epilepsy have been evaluated in relation to the AED as well as in relation to the time of epilepsy, but the cognitive level among patients with primary non-affected epilepsy in cognition (temporal lobe epilepsy, temporal mesial, JME) has not been compared to their siblings who live in the same environment and have the same opportunities for education.

\section{Justification}

It is estimated that in Mexico, 1 to $2 \%$ of the population suffers from epilepsy. Little is known about this population in terms of employment and quality of life in general. In addition to the quality of life, there is a stigma towards patients with epilepsy that leads the patient not to look for work, not attend school and finally, not to be part of the productive society of the country. Few studies establish the cognitive difference between patients with epilepsy diagnosis and people without epilepsy. The total of the studies found are comparisons between randomized and paired groups, without taking into account the psychosocial environment in which the individuals or consanguinity developed. No studies have compared cognitive areas of language, attention, memory, calculus, and orientation between siblings, one with epilepsy and the other healthy. Therefore it was proposed to investigate and study siblings, one healthy and one with epilepsy since in our country, the environment where people develop is essential in terms of the development of the cognition. With the results obtained in this study, we will have statistical support to propose to the state and the national health systems to integrate the patients with FEUE and JME into the productive society, as well as to advise families on the intellectual abilities of their patients.

\section{Objectives}

1. Compare the cognitive status between epileptic patients and a healthy brother.

2. Evaluate and compare cognitive status between a group of epileptic patients and healthy subjects as a control group. 
3. Correlate the cognitive state among epileptic patients according to management with monotherapy or polytherapy.

\section{Hypothesis}

- There is a significant difference in the cognitive area between epileptic patients and their siblings in one of the following areas: attention, memory, and calculation.

- There is a significant cognitive difference between epileptic patients and healthy controls in the areas of care, memory, and calculation.

- $\quad$ There is no cognitive difference in a group of people with epilepsy concerning the use of monotherapy and polytherapy.

\section{Design}

\section{Type of study}

An analytical, case-control, cross-sectional, descriptive, observational study.

\section{Universe}

Patients with FEUE and JME in the state of San Luis Potosi, Mexico.

\section{Sample}

The sample was taken for convenience during November according to the Neurology consultation of the Central Hospital, and that meet the inclusion and exclusion criteria. A total of 30 epileptic patients and 30 healthy siblings were included.

\section{Inclusion criteria}

- $\quad$ Patients with FEUE and JME.

- Patients with monotherapy treatment: Carbamazepine, Phenytoin, Valproate, Levetiracetam, Lamotrigine.

- Patients with polytherapy treatment with any combination of two medications: Carbamazepine, Phenytoin, Valproate, Levetiracetam, Lamotrigine.

- $\quad$ Age over 12 years and under 65 years.

- $\quad$ Brothers with + or - 3 years difference

\section{Exclusion criteria}

- $\quad$ Patients with epilepsy secondary to neonatal hypoxia.

- Subjects diagnosed with a pervasive developmental disorder.

- Patients with structural lesions at the frontal or temporal level.

- $\quad$ Patients with symptomatic epilepsy or secondary to infectious processes.

- $\quad$ Illiterate subjects.

- Patients with medication use other than those in the study (Phenobarbital, primidone, topiramate have been linked to changes in cognition).
- Patients who do not sign an informed consent.

\section{Elimination criteria}

- Participants voluntarily decide to suspend their performance during the CSID test.

\section{Variables}

\section{Independent}

- Age: Registered as the number of years in the sociodemographic questionnaire.

- Gender: Registering as male or female in the sociodemographic questionnaire.

- Diagnostic time: Registering as several years in the sociodemographic questionnaire.

- $\quad$ Drugs used as monotherapy or polytherapy: Registering the type of monotherapy drug either Carbamazepine, Phenytoin, Valproate, Levetiracetam or Lamotrigine and for combination therapy two or more of the medications described in the monotherapy item

- $\quad$ Type of epilepsy: Registering if the patient has a diagnosis of idiopathic FEUE or JME.

\section{Dependent}

- Attention: assessed by the CSID scale with an ordinal score.

- Orientation: assessed by the CSID scale with an ordinal score.

- Memory: assessed by the CSID scale with an ordinal score.

- $\quad$ Calculus: assessed by the CSID scale with an ordinal score.

- $\quad$ Praxis: assessed by the CSID scale with an ordinal score.

- Language: assessed by the CSID scale with an ordinal score.

\section{Methods}

The Community-Scale Interview for Dementia (CSID) was used in this research and an informed consent letter was provided. The instrument was applied to all patients who agreed to enter the study and to their healthy family member separately to avoid that patients or relatives could learn to respond before conducting the survey. The application was made by the principal investigator and two sub-investigators trained by the principal investigator. With the demographic file and the solved instrument, the data was captured in the Excel software to obtain results and analysis. For the statistical analysis, measures of central tendency, percentages, Mcnemar test, contingency tables, hypothesis test, student T were used to be able to determine the difference between the groups demographically and the differences between the scores obtained in all the items of the CSIDs that included orientation, memory, praxis, attention and calculation, and language. The significance level was $\mathrm{p}<0.05$. 
The Community-Scale Interview For Dementia is an instrument designed to define the items of memory, praxis, language, orientation, and attention and calculation for screening for dementia also used to assess the items described independently. The scale has 45 items, and the total scores were compared with epileptic patients and their healthy family in the first analysis, then two groups were formed, one of the patients with epilepsy and another of patients without epilepsy and they were analyzed. When grouping patients with epilepsy, patients were divided by monotherapy and polytherapy management, the scores obtained in each item of the scale were compared.

\section{Results}

Thirty-three patients with epilepsy were captured in the Neurology Service of the "Ignacio Morones Prieto" Central Hospital and the private consultation of the thesis advisor. It was ensured that the patients came with a brother or sister of +-3 years apart and that they met the pre-established criteria for the investigation. Three patients were excluded because they did not meet the inclusion and exclusion criteria or because they did not show up with a healthy family member.

\section{Age}

Data analysis revealed that the average age in patients with epilepsy was 27.9 years, with a standard deviation of 11.75 . In the group of healthy siblings, the average age was 28.6 years with a standard deviation of 11.58. When applying the McNemar test between epileptic subjects and their relatives, a $\mathrm{P}=0.59$ was obtained, this difference is statistically not significant.

\section{Gender}

Data analysis revealed that the gender distribution in the group of people with epilepsy was 14 women and 16 men; in the group of healthy siblings, it was 23 women and seven men. The X2 was 3.76 with a $\mathrm{P}=0.05$, statistically significant.

\section{Type of epilepsy}

Among the 30 subjects with epilepsy (cases), it was found that 17 were diagnosed with FEUE and 13 with JME.

\section{Years with the diagnosis of epilepsy}

Patients with epilepsy were grouped into two groups: those with 9 or fewer years since the diagnosis (8 patients) and those with 10 or more years since the diagnosis (22 patients).

\section{Treatment of epilepsy}

Eight patients were receiving monotherapy, while 22 patients had polytherapy.

\section{Comparison of Cognoscitive Areas}

When comparing the items of memory, language, orientation, praxis, attention, and calculation in pairs made up of an epileptic patient and his healthy brother or sister, the results that were found are summarized in Table 1.

Table 1: Comparison between patients and their siblings in the CSID cognitive areas.

\begin{tabular}{|c|c|c|c|c|}
\hline Cognitive Area & Average Difference & Differences' Standard Deviation & $\mathbf{t}_{\mathrm{n}-2}$ & 1.38 \\
\hline Language & 1.7 & 6.74 & 0.18 (NS) & 0.13 \\
\hline Memory & -0.13 & 5.67 & -0.3 \\
\hline Orientation & -0.07 & 1.2 & 0.76 (NS) \\
\hline Attention and calculation & 0 & 0.455 & 1 \\
\hline Praxis & -0.2 & 0.714 & -1.53 \\
\hline
\end{tabular}

In the memory, orientation, and praxis areas, the averages obtained were negative because the average was calculated by subtracting the result of epileptic patients from healthy patients. Since the established hypothesis was that healthy subjects should have a higher score than patients with epilepsy, surprisingly, when applying the statistical test, it was found in all the items analyzed that there was no statistically significant difference. When making the comparison in the areas of memory, language, orientation, praxis, attention and, calculation using the global epileptic patients and healthy subjects that were demographically equal by age and schooling, the results described in Table 2 were found (Table 2).

Table 2: Comparison between patients with epilepsy (cases) and the group with healthy subjects (controls) in the CSID cognitive areas.

\begin{tabular}{|c|c|c|c|c|c|c|}
\hline \multirow{2}{*}{ Cognitive area } & \multicolumn{2}{|c|}{ Average } & \multicolumn{2}{|c|}{ Standard deviation } & \multirow{2}{*}{$t_{n 1+n 2-2}$} & \multirow{2}{*}{$p$} \\
\hline & Case & Control & Case & Control & & \\
\hline Language & 34.2 & 35.9 & 5.36 & 7.39 & -1.00 & $0.32(\mathrm{NS})$ \\
\hline Memory & 37.6 & 37.4 & 6.31 & 6.18 & 0.08 & 0.94 (NS) \\
\hline Orientation & 8.733 & 8.67 & 1.31 & 1.32 & 0.19 & 0.85 (NS) \\
\hline $\begin{array}{l}\text { Attention and } \\
\text { calculation }\end{array}$ & 5.8 & 5.8 & 0.54 & 0.4 & 0 & 1 (NS) \\
\hline Praxis & 2 & 1.8 & 0.68 & 0.6 & 1.18 & $0.24(\mathrm{NS})$ \\
\hline
\end{tabular}

The $\mathrm{p}$ was not significant in the five items analyzed; therefore, overall, there was no difference between the two groups regardless of consanguinity and the educational, cultural, and social environment.
The group of 30 patients with epilepsy was taken and divided according to management with monotherapy or polytherapy to analyze if there was any statistically significant difference in any item of the scale applied, the results are shown in Table 3. 
Table 3: Comparison in cognitive areas in the group of epileptic patients subdividing them in those with monotherapy (Mono) vs. polytherapy (Pol).

\begin{tabular}{|c|c|c|c|c|c|c|}
\hline \multirow{2}{*}{ Cognitive area } & \multicolumn{2}{|c|}{ Average } & \multicolumn{2}{c|}{ Standard deviation } & \multirow{2}{*}{$\mathbf{t}_{\text {n1+n2-2 }}$} & \multirow{2}{*}{$\boldsymbol{p}$} \\
\cline { 2 - 6 } & Pol. & Mono. & Pol. & Mono. & -1.25 & 0.22 (NS) \\
\hline Language & 33.45 & 36.25 & 5.32 & 4.92 & -0.83 & 0.41 (NS) \\
\hline Memory & 36.59 & 39 & 6.87 & 6.63 & -1.63 & 0.11 (NS) \\
\hline Orientation & 8.5 & 9.38 & 1.41 & 0.7 & -0.44 & 0.66 (NS) \\
\hline $\begin{array}{c}\text { Attention and } \\
\text { calculation }\end{array}$ & 5.77 & 5.88 & 0.6 & 0.33 & -1.2 & 0.24 (NS) \\
\hline Praxis & 1.91 & 2.25 & 0.67 & 0.66 & & \multirow{2}{*}{ (N) } \\
\hline
\end{tabular}

When the groups were divided by management, and each cognitive item was analyzed, it was found that no result was statistically significant when performing the hypothesis test. Additionally, the division made by the time of evolution of the epileptic patients was used to analyze all the cognitive items evaluated concerning to whether the patient had equal or less than nine years of evolution and 10 or more years of evolution of epilepsy. These results are shown in Table 4.

Table 4. Comparison of cognitive items in people with epilepsy with less than 9 and 10 or more years of diagnosis of epilepsy.

\begin{tabular}{|c|c|c|c|c|c|c|}
\hline \multirow{2}{*}{ Cognitive area } & \multicolumn{2}{|c|}{ Average } & \multicolumn{2}{|c|}{ Standard deviation } & \multirow{2}{*}{$t_{n 1+n 2-2}$} & \multirow{2}{*}{$p$} \\
\hline & $\geq 10$ & $\leq 9$ & $\geq 10$ & $\leq 9$ & & \\
\hline Language & 34.27 & 34 & 5.36 & 5.36 & 0.11 & $0.91(\mathrm{NS})$ \\
\hline Memory & 38.045 & 35 & 6.88 & 6.38 & 1.05 & $0.30(\mathrm{NS})$ \\
\hline Orientation & 8.6818 & 8.875 & 1.22 & 1.53 & -0.34 & $0.73(\mathrm{NS})$ \\
\hline $\begin{array}{l}\text { Attention and } \\
\text { calculation }\end{array}$ & 5.8636 & 5.625 & 0.45 & 0.69 & 1.05 & $0.30(\mathrm{NS})$ \\
\hline Praxis & 2 & 2 & 0.6 & 0.86 & 0 & $1(\mathrm{NS})$ \\
\hline
\end{tabular}

There were no statistically significant differences in any item evaluated by the CSID scale in the time groups of the disease.

\section{Analysis}

For the analysis of this research, 30 couples were studied, consisting of a patient diagnosed with FEUE or JME and a healthy control brother or sister with a +-3 years difference in their age. Controls were obtained for convenience, regardless of gender.

\section{Demographic data}

When performing the analysis with the McNemar test, the $\mathrm{p}$ was 0.59 , which is statistically not significant. This analysis was performed to support that the groups were demographically similar in terms of age and therefore, comparable. Regarding gender, for reasons of the sample, it was decided to include couples interchangeably since the sample was significantly reduced by matching the groups formed by gender. This analysis revealed that the difference was statistically significant with a p of 0.05 , however, in the literature there is no mention of different results in the CSID test for gender, so it was considered that this difference did not affect the analysis of the results obtained for the stated objectives. Regarding the degree of study, the CSID Test established that one of the advantages when applying the test is that it can be used in subjects of different social strata and degree of study, with the only condition being that the patient can read and write, and there is less attended some grade of primary education [43]. 100\% of the subjects included in the study complied with this provision.

\section{CSID test analysis}

To make a first analysis, the participants were taken as distinctively shaped partners as previously described, obtaining a score subtracting the value obtained in the test by the patient with epilepsy to the outcome of the control subject, given that the studies and the bibliographic support states that there are better test scores in healthy subjects on patients with epilepsy $[10,17,22,27,28,29,34]$. Table 1 shows the differences of these averages, highlighting that in the language area, a difference of 1.7 was met in favor of healthy subjects of; In the area of attention and calculation there was no difference. It is noteworthy that in the areas of memory, orientation, and praxis, it was a difference in the averages of $-0.13,-0.07$ and -0.02 , respectively. This differs from the data reported in studies that make a comparison between cases and controls and where it is established that epileptic patients have a lower score with statistical significance in the memory items [17,9,20,22,34] and language [44,24,34] (one author even associates benign centrotemporal points specifically to alterations in language [45]), attention and calculation [1,34,46] and finally praxis [34,47]. It has been suggested that apraxia is due to visuospatial and visuoconstructional dysfunction of patients with epilepsy [47].

In the study subjects, the cases had a better score than the controls, however statistically, these differences are not significant. Even this result is different from what was reported in the literature, and contradicts the initial alternative hypothesis for this investigation, which makes it more interesting, in addition to positive to establish that there is no difference between siblings when we compare subjects with epilepsy with respect to healthy controls in all the analyzed areas, which shows that the epileptic patient is potentially productive for society at least in the activities carried out by his healthy brother. In a secondary analysis, only two groups were taken regardless of the specific partner, comparing the 
group of epileptic patients vs. the group of healthy controls globally regardless of their blood relationship. In this analysis (as shown in Table 2), the same trend that was found in the analysis in pairs was reproduced, obtaining once again higher scores in epileptic patients in the areas of memory, orientation and praxis, with a difference of $-0.2,-0.05,-0.2$, respectively.

All these results were once again statistically non-significant, contradicting the established hypothesis and demonstrating that, if it is taken as independent groups, there is no difference in the items analyzed. A third analysis was performed in a group of 30 patients with epilepsy. It was found that it was made up of 17 patients with a diagnosis of FEUE and 13 with a diagnosis of JME. These patients were divided into two groups based on the treatment received: as a monotherapy group and a polytherapy group. The first being formed by eight patients and the second, by 22 patients. When comparing the results (Table 3), no statistically significant differences were found in the analyzed items. There are reports in the literature that establishes that the medications used in the inclusion criteria of this study do not affect memory, language, calculus, attention, or praxis [32-40]. Therefore, these results support the hypothesis that there would be no difference in the cognitive items evaluated by the CSID with the management of the selected medications.

The patients with epilepsy were divided into two groups according to the time of diagnosis: the first, included patients with 9 or fewer years of diagnosis, with a total of 17 cases, and the second group included patients with 10 or more years of diagnosis of epilepsy, in which the remaining 13 patients were classified. The patients were subjected to the hypothesis test obtaining in all cases, a p > of 0.05 being non-statistically significant, as shown in Table 4 .

In the literature, it has been suggested that, especially in FEUE involving the temporal lobe and Hippocampal regions, there may have a more considerable deterioration according to the time of evolution of epilepsy, established as a lower performance at ten years of diagnosis [15-20,22,28,30,31]. Our results did not show a difference between the patients and their controls, according to the time of evolution. This means that among the cases of epilepsy analyzed, there was no statistically significant difference in terms of time.

\section{Conclusions}

1. When analyzing the cases and controls studied, there was no statistically significant difference in the areas of memory, praxis, language, orientation, attention, and calculation when comparing the epileptic patient with his healthy brother, performing the analysis in pairs.

2. In this case-control study, no statistically significant difference was established in the areas of memory, praxis, language, orientation, care, and calculation when comparing the groups of epileptic patients with the group of healthy controls. When comparing epileptic patients who receive monotherapy treatment to those who receive polytherapy treatment, there is no statistically significant difference in the areas of memory, praxis, language, orientation, care, and calculation.
3. By sub-grouping epileptic patients based on the evolution time since the diagnosis of epilepsy in those who have 9 or less years against those with 10 or more years, there is no statistically significant difference in the areas of memory, praxis, language, orientation, care and calculation, so the chronicity of the condition does not seem to influence the cognition of the patients with epilepsy.

\section{Comments}

These results demonstrate that, in the absence of a difference in cognitive status between patients with FEUE and JME, subjects can be economical, labor, and culturally active in society.

The study was limited to a single population, so it is suggested that the same research should be carried out in a multicenter manner in order to apply the same conclusions throughout the country. It is suggested to add an additional test for the calculation assessment, which in the CSID may be limited.

\section{Ethical Considerations and Funding}

No ethical considerations to declare. The funding of this study was internal.

\section{Acknowledgement}

None.

\section{Conflict of Interest}

No conflict of interest.

\section{References}

1. (2007) CONTINUUM: Lifelong Learning in Neurology. Epilepsy 13(4): 13.

2. Shorvon SD, Perucca E, Engel J (2009) The Treatment of Epilepsy. Chichester, Wiley-Blackwell, UK, pp. 20-22.

3. Rubio F Reséndiz JC, Sentíes H Alonso M (2000) Epilepsia. Programa prioritario de epilepsia. pp. 7-10

4. Nicoletti A, Reggio A, Bartoloni A, Failla G, Sofia V, et al. (1999) Prevalence of epilepsy in rural Bolivia A door-to-door survey. Neurology 53(9): 2064-2069.

5. Scheffer IE, Berkovic S, Capovilla G, Connolly MB, French J, et al. (2017) ILAE classification of the epilepsies: Position paper of the ILAE Commission for the Classification and Terminology. Epilepsia 58(4): 512-521.

6. Iinuma K, Morimoto K, Akiyama T, Ikeda A, Kurihara M (2006) Proposed diagnostic scheme for the classification of epileptic seizures and epilepsies (ILAE, 2001): proposal from Japan Epilepsy Society. Epilepsia 47(9): 1588-1589.

7. (2010) CONTINUUM: Lifelong Learning in Neurology. Epilepsy 16(3): 15-20.

8. Wu DY, Ding D, Wang Y, Hong Z (2010) Quality of life and related factors in Chinese adolescents with active epilepsy. Epilepsy Res 90(1-2): 16-20.

9. Besag F (1995) Epilepsy, Learning and Behavior in childhood. Epilepsia 36(sup 1): s58-s63.

10. McCagh J, Fisk JE, Baker GA (2009) Epilepsy, psychosocial and cognitive functioning. Epilepsy Res 86(1): 1-14.

11. Fisher RS, Vickrey BG, Gibson P, Hermann B, Penovich P, et al. (2000) The impact of epilepsy from the patient's perspective. Views about therapy and health care. Epilepsy Res 41: 53-61.

12. (2003) International League Against Epilepsy, Quality of life: general considerations. Epilepsia 44(Suppl. 6): 58-158. 
13. Baxendale S, O’Toole A (2007) Epilepsy myths: alive and foaming in the $21^{\text {st }}$ century. Epilepsy Behav 11: 192-196.

14. Ryan R, Kempner K, Emlen AC (1980) The stigma of epilepsy as a selfconcept. Epilepsia 21: 433-444.

15. Mensah SA, Beavis JM, Thapar AK, Kerr MP (2007) A community study of the presence of anxiety order in people with epilepsy. Epilepsy Behav 7: 438-446.

16. Helmstaedter C (2001) Behavioural aspects of frontal lobe epilepsy. Epilepsy Behav 2: 384-395.

17. Taylor J, Kolamunnage-Dona R, Marson AG, Smith PE, Aldenkamp AP, et al. (2010) Patients with epilepsy: cognitively compromised before the start of antiepileptic drug treatment? Epilepsia 51(1): 48-56.

18. De Boer HM, Mula M, Sander JW (2008) The global burden and stigma of epilepsy. Epilepsy Behav 12: 540-546.

19. Hendriks MPH, Aldenkamp AP, van der Vlugt H, Alpherts WCJ, Vermeulen J (2002) Memory complaints in medically refractory epilepsy: relationship to epilepsy-related factors. Epilepsy Behav 3: 165-172.

20. Aldenkamp AP, Bodde N (2005) Behaviour, cognition and epilepsy. Acta Neurol Scand 112 (s182): 19-25.

21. Hermann B, Jones J, Sheth R, Dow C, Koehn M, et al. (2006) Children with new-onset epilepsy: neuropsychological status and brain structure. Brain 129: 2609-2619.

22. Sonmez F, Atakli D, Sari H, Atay T, Arpaci B (2004) Cognitive function in juvenile myoclonic epilepsy. Epilepsy Behav 5(3): 329-336.

23. Mathiak KA, Mathiak K, Wolańczyk T, Ostaszewski P (2009) Psychosocial impairments in children with epilepsy depend on the side of the focus. Epilepsy Behav 16(4): 603-608.

24. Giordani B, Caveney AF, Laughrin D, Huffman JL, Berent S, et al. (2006) Cognition and behavior in children with benign epilepsy with centrotemporal spikes (BECTS). Epilepsy Res 70(1): 89-94.

25. Haut SR, Katz M, Masur J, Lipton RB (2009) Seizures in the elderly: impact on mental status, mood, and sleep. Epilepsy Behav 14(3): 540544 .

26. Besag FM (2006) Cognitive and behavioral outcomes of epileptic syndromes: implications for education and clinical practice. Epilepsia 47 Suppl 2:119-25.

27. Cooper AD, Britton JW, Rabinstein AA (2009) Functional and cognitive outcome in prolonged refractory status epilepticus. Arch Neurol 66(12): 1505-1509.

28. Motamedi G, Meador K (2003) Epilepsy and cognition. Epilepsy Behav 4 Suppl 2: S25-38.

29. Carreño M, Donaire A, Sánchez-Carpintero R (2008) Sánchez-Carpintero R. Cognitive disorders associated with epilepsy: diagnosis and treatment. Neurologist 14(6): S26-34.

30. Bell BB, Hermann BP, Woodard AR, Jones JE, Rutecki PA, et al. (2001) Object naming and semantic knowledge in temporal lobe epilepsy. Neuropsychology 15(4): 434-443.
31. Moore PM, Baker GA (2002) The neuropsychological and emotional consequences of living with intractable temporal lobe epilepsy: implications for clinical management. Seizure 11(4): 224-230.

32. Farrant A, Morris RG, Russell T, Elwes R, Akanuma N, et al. (2005) Social cognition in frontal lobe epilepsy. Epilepsy Behav 7: 506-516.

33. Meador KJ, Loring DW, Huh K, Gallagher BB, King DW (1990) Comparative cognitive effects of anticonvulsants. Neurology 40: 391-394.

34. Sunmonu TA, Komolafe MA, Ogunrin AO, Ogunniyi A (2009) Cognitive assessment in patients with epilepsy using the Community Screening Interview for Dementia. Epilepsy Behav 14(3): 535-539.

35. Aldenkamp AP, Arends J, Bootsma HP, Diepman L, Hulsman J, et al. (2002) Randomized double-blind parallel-group study comparing cognitive effects of a low-dose lamotrigine with valproate and placebo in healthy volunteers. Epilepsia 43: 19-26.

36. Lamberty Y, Margineanu DG, Klitgaard H (2000) Absence of negative impact of levetiracetam on cognitive function and memory in normal and amygdala-kindled rats. Epilepsy Behav 1: 333-342.

37. Kossoff EH, Bergey GK, Freeman JM, Vining EP (2001) Levetiracetam psychosis in children with epilepsy. Epilepsia 42: 1611-1613.

38. Tatum WO, French JA, Faught E, Morris GL, Liporace J, et al. (2001) Postmarketing experience with topiramate and cognition. Epilepsia 42(9): 1134-1140.

39. Iqbal N, Caswell HL, Hare DJ, Pilkington O, Mercer S, et al. (2009) Neuropsychological profiles of patients with juvenile myoclonic epilepsy and their siblings: a preliminary controlled experimental video-EEG case series. Epilepsy Behav 14(3): 516-521.

40. Buelow JM, McNelis A (2002) Should every child with epilepsy undergo a neuropsychological evaluation? Epilepsy Behav 3(3): 210-213.

41. Prince M, Ferri CP, Acosta D, Albanese E, Arizaga R, et al. (2007) The protocols for the 10/66 dementia research group population-based research programme. BMC Public Health 20(7): 165.

42. Shin HY, Chung EK, Rhee JA, Yoon JS, Kim JM (2005) Prevalence and related factors of dementia in an urban elderly population using a new screening method. J Prev Med Public Health 38(3): 351-358.

43. Hall K, Gao S, Emsley C, Ogunniyi A, Morgan O, et al. (2000) Community screening interview for dementia (CSID); performance in five disparate study sites. Int J Geriatr Psychiatry 15: 521-531.

44. Parkinson GM (2002) High incidence of language disorder in children with focalepilepsies. Dev Med Child Neurol 44: 533-537.

45. Monjauze C, Tuller L, Hommet C, Barthez MA, Khomsi A (2005) Language in benign childhood epilepsy with centro-temporal spikes. Brain Lang 92: 300-308.s

46. Huang CW, Itsieh YJ, Tsai JJ, Pai MC (2005) Cognitive performance in cryptogenic epilepsy. Acta Neurol Scand 112: 228-233.

47. Volki-Kernstock S, Willinger U, Feucht M (2006) Special perception and spatial memory in children with benign childhood epilepsy with centrotemporal spikes (BCECTS). Epilepsy Res 72: 39-48. 